\title{
Depresión y ansiedad en personas con retinosis pigmentaria, sus familiares y un grupo control ${ }^{*}$
}

Depression and anxiety in people with retinitis pigmentosa, their family and a control group

Recibido: octubre 22 de 2010 | Revisado: marzo 23 de 2011 | Aceptado: marzo 26 de 2011

\author{
M. DOLORES LÓPEZ-JUSTICIA ** \\ Facultad de Psicología, Universidad de Granada, España \\ TAMARA POLO SÁNCHEZ ${ }^{* * *}$ \\ CAROLINA FernáNDEZ JiMÉnEZ \\ HELENA CHACÓN LÓPEZ \\ Facultad de Ciencias de la Educación. \\ Universidad de Granada, España \\ Carmen Díaz Batanero* \\ Facultad de Ciencias de la Educación, \\ Universidad de Huelva, España \\ Antonio Chacón Medina* \\ Facultad de Ciencias de la Educación, \\ Universidad de Granada, España
}

SICI: 1657-9267(201108) 10:2<467:DAEPRP>2.0.TX;2-K

Para citar este artículo. López-Justicia, M. D., Polo, S. T., Chacón, L. H., Díaz, B. C. \& Chacón, M. A. (2011). Depresión y ansiedad en personas con retinosis pigmentaria, sus familiares y un grupo control. Universitas Psychologica, 10 (2), 467-476.

* Artículo de investigación.

** Dpto. de Psicología Evolutiva y de la Educación. Universidad de Granada. Facultad de Psicología. Campus de Cartuja. 18071, Granada (España). Email:dlopezj@ugr.es

*** Dpto. de Psicología Evolutiva y Educación. Facultad de Ciencias de la Educación. Universidad de Granada. Campus de Cartuja. 18071, Granada (España). E-mails: tpolo@ugr.es; carolina@ugr.es; helbeta@ correo.ugres

***** Dpto. de Psicología Clínica, Experimental y Social. Facultad de Ciencias de la Educación. Universidad de Huelva. Campus del Carmen, Av. de las Fuerzas Armadas, 21071, Huelva (España). E-mail: carmen. diaz@dpsi.uhu.es

******* Dpto. de Didáctica y Organización Escolar. Facultad de Ciencias de la Educación. Universidad de Granada. Campus de Cartuja. 18071, Granada (España).E-mail: chacon@ugr.es

\section{RESUMEN}

El estudio pretendió determinar la presencia de depresión y/o ansiedad en adultos con retinosis pigmentaria (RP) y en sus familiares, frente a un grupo control. También trató de averiguar si la agudeza visual (AV) de los afectados tenía relación con estas variables. Se utilizó el Cuestionario de Ansiedad STAI y el Inventario de Depresión de Beck. Los resultados mostraron depresión leve en los afectados y mayores niveles de ansiedad, como estado y como rasgo, tanto en ellos como en sus familiares; aunque no se encontró relación con la AV. Se recomienda evaluar la ansiedad y la depresión tras el diagnóstico de la enfermedad y transcurrido un tiempo, para proporcionar apoyo psicológico adecuado a los afectados y a los familiares.

Palabras clave autores

Depresión, ansiedad, agudeza visual, retinosis pigmentaria, adultos.

Palabras clave descriptores

Psicología experimental, ciencias del comportamiento, psicología clínica, test psicológico, psicoanálisis, psicoterapia.

\section{A B S T R A C T}

The aim of this study was to determine the possible presence of depression and / or anxiety in adults with Retinitis Pigmentosa (RP), as well as their families, compared to a control group. It was also intended to determine if the Visual Acuity (VA) have any impact on the variables analyzed. It was used the State - Trait Anxiety Inventory and the Beck Depression Inventory. Results showed that anxiety was higher in people with RP and their families, as a state and as a feature, and the presence of mild depression in RP group. No relationship was found between VA and these variables. It is recommended to evaluate anxiety and depression variables just after the diagnosis of the disease and again over time, in order to provide psychological support to those affected and their families.

Key words authors

Depression, Anxiety, Visual Acuity, Retinitis Pigmentosa, Adults.

Key words plus

Experimental Psychology, Behavioural Sciences, Clinical Psychology, Psychological Tests, Psychoanalysis, Psychotherapy. 


\section{Introducción}

La retinosis pigmentaria $(\mathrm{RP})$ pertenece a un grupo de enfermedades degenerativas de retina caracterizadas por una pérdida progresiva de la visión que, con frecuencia, puede conducir a la ceguera (Fernández, 2007). Consiste en la degeneración de los fotorreceptores (conos y bastones) retinianos, dando lugar a áreas incapaces de responder a los estímulos luminosos. Los síntomas y grados de afectación son diversos, siendo los más habituales la pérdida de visión periférica o central, la dificultad para ver de noche o en penumbra, la dificultad de adaptación a los cambios de luz, o alteraciones en la discriminación de colores (Geruschat \& Turano, 2002; Rundquist, 2004). Afecta aproximadamente a 1 de cada 3.700 personas y es más común en hombres (65\%) que en mujeres (55\%) (Fernández, 2007).

Es una enfermedad congénita cuya progresión, y el grado de discapacidad que lleva asociado, puede provocar grandes cambios educativos, sociales y laborales en la vida de los afectados (Nemshick, Vernon \& Ludman, 1986).

Con frecuencia muchos afectados dan respuestas típicas tras el diagnóstico, como: aislamiento social, retraimiento, abandono de los estudios o del trabajo, síntomas depresivos, ansiedad, alteraciones en su autoconcepto, etc. (López-Justicia \& Pichardo, 2001; Sacks, Wolffe \& Tierney, 1998); pero estas reacciones varían en función de la percepción que tengan de su enfermedad, de los recursos personales y sociales y del control que ejerzan sobre los acontecimientos que les afectan (Wahl, Becker, Burmedi \& Schilling, 2004).

La depresión es uno de los trastornos más comunes en jóvenes y adultos, con tendencia a la cronicidad y con un alto coste personal, económico y social. El riesgo de presentarla es mayor en grupos con especiales características, lo que justificaría la puesta en práctica de medidas preventivas ante la presencia de un alto número de síntomas depresivos, aun no llegando al criterio de depresión (Vázquez \& Torres, 2007). Se ha estimado que su prevalencia en afectados por RP es del 25.7 \% (mientras que en la población general es del $10 \%$ ), cifra similar a la de otras enfermedades crónicas como la diabetes, el infarto de miocardio y el cáncer (Hahm, Shin, Shim, Jeon, Seo, Cheng $\&$ Yu, 2008).

Desde la teoría cognitiva de depresión de Beck (1987) se considera que la principal alteración que se produce en la depresión se encuentra en el procesamiento de la información. El factor de riesgo que hace vulnerable a la depresión es la posesión de una serie de esquemas desadaptativos, con contenido negativo de pérdida y fracaso. Estos suelen adquirirse en la infancia, aunque pueden permanecer latentes y activarse cuando la persona vive situaciones estresantes o sucesos ambientales específicos. El contenido de estos esquemas lo constituye la "triada cognitiva", que incluye una visión negativa de sí mismo, del mundo y del futuro, cuya acción en el procesamiento de la información lleva a una percepción distorsionada de la realidad, por lo que la persona deprimida está convencida de que las cosas son tan negativas como las percibe.

Por otra parte, la ansiedad es la anticipación aprensiva de un daño o desgracia futuros, acompañada de un sentimiento de disforia o de síntomas somáticos de tensión, pudiendo ser el objetivo del daño anticipado interno o externo (López-Ibor \& Valdés, 2002). Cuando se alude a la ansiedad, se debe diferenciar entre estado y rasgo (Spielberger, Gorsuch \& Lushene, 2002), el primero se refiere a un estado o condición emocional transitoria, caracterizada por sentimientos subjetivos de tensión, aprensión e hiperactividad del sistema nervioso autónomo; mientras que la ansiedad rasgo señala una propensión ansiosa estable o una tendencia a percibir las situaciones como amenazadoras, que eleva la ansiedad estado. Altas puntuaciones en ansiedad predicen problemas emocionales que acompañan a determinadas patologías y se suele asociar, entre otros rasgos, a depresión (Páez, Jofré, Azpiroz \& De Bortoli, 2009; Sánchez, Aparicio \& Dresch, 2006).

Distintos autores (Hahm et al., 2008; Mogk, Riddering, Dahl, Bruce \& Bradford, 2000) han señalado que las personas adultas que adquieren la restricción visual, tienen más riesgo de sufrir depresiones, lo que parece deberse a su percepción de 
pérdida de habilidad para desempeñar tareas de la vida diaria, de la creencia de ser una carga para sus familias y su dependencia para realizar otras tareas domésticas, o de desenvolvimiento en el espacio, pues, en muchas ocasiones, actividades como estas se ven seriamente dañadas (Geruschat \& Turano, 2002; Rundquist, 2004). De hecho, parece que es la pérdida funcional más que el daño en sí mismo lo que produce mayor dolor o sufrimiento (Zeiss, Lewinsohn, Rohde \& Seeley, 1996). Otros estudios han señalado que las personas que tienen un bajo sentido de autoeficacia parecen tener más riesgo de depresión (Horowitz \& Reinhardt, 2000), mientras que las que mantienen un sentimiento de control sobre su condición visual experimentan menor deterioro funcional (Wahl et al., 2004).

Estados psicológicos negativos como ansiedad y depresión aparecen asociados a la RP, como han puesto de relieve los estudios de Hahm et al. (2008) y de Strougo, Badoux y Duchanel (1997). El primer estudio concluía que el alto riesgo de depresión que tienen, incide de forma negativa en el uso de su visión y reduce su calidad de vida, independientemente de la condición demográfica, función visual objetiva y gravedad de la enfermedad. Mientras que el segundo, señalaba que las mujeres afectadas por RP que se sentían más dolidas o se percibían inferiores a los demás, presentaban más conductas fóbicas y mayores niveles de ansiedad y depresión que los hombres.

La pérdida de visión en la RP es impredecible, inexorable y su progreso conlleva una pérdida de habilidad para realizar determinadas tareas, menoscabando la autoconfianza y autocontrol de los afectados, pudiendo conducirlos a percibirse de forma más negativa (Kiser \& Dagnelie, 2008). Sin embargo, en este proceso hay agentes sociales, como la familia, cuyo apoyo o ayuda favorecen la adaptación a la pérdida de la visión y mejoran su calidad de vida (Reinhardt, 2001). En este sentido, Nemshick et al. (1986) subrayaban que un porcentaje muy alto de afectados consideraba que el apoyo proporcionado por la familia era alto, mientras que otros percibían sobreprotección y se quejaban de que sus familiares no comprendían su condición, ignoraban o negaban el problema; por ello, sugerían que también recibieran ayuda, ya que las consecuencias de la RP los afectaban, igualmente. Lo que parece evidente es que las familias deben conocer los cambios producidos en los afectados (asociados al carácter degenerativo de la enfermedad), las capacidades que mantienen, evitar actitudes sobreprotectoras y saber qué hacer, cuándo y cómo para no interferir en sus decisiones (Cimarolli \& Boerner, 2005; López-Justicia \& Nieto, 2006).

Otras investigaciones han tratado de conocer en qué medida podría estar influyendo la cifra de agudeza visual (AV), o capacidad para discriminar detalles finos (López-Justicia, 2004), en la presencia de depresión y ansiedad en personas con dificultades visuales, sobre todo con degeneración macular asociada a la edad (Augustin et al., 2007; Lee, Gómez-Marín \& Lam, 2000), observando que la depresión era más severa en personas con menor AV, mientras que la ansiedad no estaba relacionada con esta.

A pesar de que son escasos los estudios que analizan el efecto de la RP en el ajuste psicosocial de las personas afectadas, sí parece evidente que la depresión y la ansiedad son comunes aunque, de acuerdo con Nemshick et al. (1986), el periodo de mayor crisis o estrés se produce durante, o inmediatamente después del diagnóstico. También es conocido que las enfermedades crónicas repercuten no solo en el bienestar y calidad de vida de los afectados, sino en sus allegados (Agudelo, Casadiegos \& Sánchez, 2009), pudiendo hacerles más vulnerables a la depresión y/o la ansiedad. Asimismo, como se ha puesto de relieve, otras investigaciones han subrayado que la depresión incrementa la probabilidad de deterioro del uso efectivo de la visión, lo que justificaría la conveniencia de evaluar estas variables e incidir en su tratamiento, en caso de requerirlo. Teniendo en cuenta los datos y aportaciones de trabajos previos, los objetivos del presente estudio fueron: en primer lugar, detectar la posible presencia de depresión y/o ansiedad en adultos afectados por RP, así como en sus familiares. En segundo lugar, investigar la relación entre estas variables y la AV en personas con RP. Este tipo de estudio intenta aportar información que permita 
orientar la intervención psicológica más oportuna en personas afectadas con RP, en vías de lograr un mayor bienestar y calidad de vida de esta población $\mathrm{y}$ de sus familiares.

\section{Método}

\section{Muestra}

Participó un total de 88 personas distribuidas del siguiente modo: un primer grupo formado por 32 afectados de RP, con una edad media de 42.5 años $(D T=11.73)$; un segundo grupo, constituido por 22 personas familiares de afectados con retinosis (edad media 43.2 años, DT $=11.25$ ); y un tercer grupo control, compuesto por 34 personas (edad media 38 años, $D T=9.22$ ) que no habían tenido contacto o relación alguna con afectados por esta enfermedad. La Tabla 1 recoge el sexo, nivel de estudios y edad de los participantes.

Las personas afectadas de RP tenían una restricción del campo visual (CV), estando comprendido entre menos de $5^{\circ}$ y $20^{\circ}$, mientras que todos presentaban una AV comprendida entre 1 y 0.05 en el mejor de sus ojos, evaluada con la escala de Wecker, siendo un criterio para la participación en esta investigación haber sido diagnosticados de la enfermedad al menos tres años antes de la evaluación y no padecer ninguna otra enfermedad o discapacidad.

Se comparó la distribución de las muestras de los tres grupos en cuanto a nivel de estudios y edad. Los grupos no difieren en cuanto al nivel de estudios $(\chi 2=13.8 ; p=0.31)$, ni en la edad $(F=$ $1.93 ; p=0.15)$. Sin embargo, no se pudo asegurar la comparación del grupo de familiares en cuanto al sexo, al estar restringido por la accesibilidad, aunque sí se realizó entre los grupos RP y control $(\chi 2=0.34 ; p=0.55)$.

\section{Procedimiento}

Para la selección de la muestra, en primer lugar se inició un contacto con la Asociación Andaluza de Retinosis Pigmentaria. Con su colaboración se organizó una reunión a la que asistieron las personas

TABLA 1

Sujetos de la muestra clasificados por sexo, nivel de estudios y edad

\begin{tabular}{lcccccccc}
\hline \multicolumn{1}{c}{ Sexo } & \multicolumn{2}{c}{ Grupo control } & \multicolumn{2}{c}{ Grupo RP } & \multicolumn{2}{c}{ Grupo Familia } & \multicolumn{2}{c}{ Total } \\
\hline Hombres & 6 & $17.6 \%$ & 4 & $12.5 \%$ & 11 & $50.0 \%$ & 10 & $15.2 \%$ \\
Mujeres & 28 & $82.4 \%$ & 28 & $87.5 \%$ & 11 & $50.0 \%$ & 56 & $84.8 \%$ \\
\hline Nivel de estudios & \multicolumn{1}{c}{ Grupo control } & \multicolumn{2}{c}{ Grupo RP } & \multicolumn{2}{c}{ Grupo Familia } & & \multicolumn{2}{c}{ Total } \\
\hline Sin estudios & 0 & - & 2 & $6.3 \%$ & 0 & - & 2 & $6.3 \%$ \\
Primarios & 13 & $38.2 \%$ & 12 & $37.6 \%$ & 10 & $45.5 \%$ & 24 & $38.0 \%$ \\
Medios & 11 & $32.4 \%$ & 6 & 18.8 & 3 & $13.6 \%$ & 17 & $25.8 \%$ \\
Superiores & 10 & $29.4 \%$ & 12 & $37.5 \%$ & 5 & $22.7 \%$ & 22 & $33.3 \%$ \\
\hline \multicolumn{1}{c}{ Edad } & Grupo control & & Grupo RP & Grupo Familia & & Total \\
\hline $20-30$ años & 8 & $23.5 \%$ & 6 & $18.8 \%$ & 2 & $9.1 \%$ & 16 & $18.2 \%$ \\
$30-40$ años & 13 & $38.2 \%$ & 7 & $21.9 \%$ & 6 & $27.3 \%$ & 26 & $29.5 \%$ \\
$40-50$ años & 7 & $20.6 \%$ & 8 & $25.0 \%$ & 9 & $40.9 \%$ & 24 & $27.3 \%$ \\
$>50$ años & 6 & $17.6 \%$ & 11 & $34.4 \%$ & 5 & $22.7 \%$ & 22 & $25.0 \%$ \\
\hline
\end{tabular}

Fuente: elaboración propia. 
afectadas y sus familiares para informarles sobre los objetivos del estudio y las actividades que se iban a realizar. Tras comunicar su deseo de participar voluntariamente en la investigación, cada afectado fue citado junto con un familiar. En un laboratorio que reunía las condiciones necesarias de luminosidad, ambos completaron las diferentes pruebas (adaptados todos los cuestionarios y hojas de respuestas a un tamaño de letra de 14 puntos, en el caso de los afectados).

Con el fin de seleccionar un grupo control se convocó a alumnos de quinto curso de Psicología para que, voluntariamente, seleccionaran y evaluaran a adultos que, distribuidos homogéneamente por edad y género, no presentaran deficiencia visual y no hubieran tenido contacto con esta problemática. Los estudiantes fueron entrenados y supervisados en la administración de las pruebas y, ante cualquier indicio de duda sobre una correcta evaluación, estas fueron rechazadas.

Todos los participantes en el estudio cumplimentaron una ficha personal, la prueba de ansiedad STAI y, posteriormente, el cuestionario de depresión de Beck. Una vez corregidos y analizados, se citó a las personas evaluadas para informarles de los resultados encontrados, ninguna puso objeción a su publicación.

\section{Instrumentos}

Para obtener información sobre edad, género y estudios, se diseñó una ficha personal, que recogía además otros datos relevantes para la investigación, tales como el año en que se diagnostica la enfermedad o la presencia de otros problemas de salud.

La evaluación de la ansiedad se realizó mediante el Cuestionario de Ansiedad Estado-Rasgo STAI (State-Trait Anxiety Inventory) (Spielberger et al., 2002), cuyo objetivo es apreciar los estados transitorios de ansiedad en el adolescente y adulto. Comprende dos escalas separadas que miden los conceptos independientes de estado (A-E) y rasgo (A-R), ambas constan de 20 ítems con 4 alternativas de respuesta que oscilan de $\mathrm{O}$ a 3 . El rango en cada una de las escalas es el mismo, de 0 a 60, aunque el punto de corte difiere: en A-R es de 25 en la población masculina y 32 en la femenina, en A-E es de 28 para la población masculina y 31 en la femenina.

La versión del STAI utilizada en el presente estudio cuenta con unos índices de consistencia interna situados entre 0.84 y 0.87 para la $\mathrm{A}-\mathrm{R}$ y entre 0.90 y 0.93 para la A-E (Spielberger et al., 2002). Los valores de fiabilidad, calculada por el procedimiento de las dos mitades, alcanzan un valor de 0.86 en la A-R y 0.94 en A-E (Spielberger et al., 2002).

Para evaluar la depresión se utilizó el Inventario de Depresión de Beck (Beck, Rush, Shaw \& Emery, 1979), un instrumento autoaplicable, validado para la población española (Vázquez \& Sanz, 1997), para cuantificar los síntomas depresivos en poblaciones normales y clínicas (Sanz \& Vázquez, 1998). El Inventario de Depresión de Beck (BDI) posee una fiabilidad media (coeficiente alfa) de 0.86 y 0.74 (Beck, Steer \& Garbin, 1988). La versión utilizada en este estudio fue la abreviada de 13 ítems, existiendo una alta correlación (0.96) entre ambas formas (Beck et al., 1979). Se eligió esta versión para facilitar la lectura a las personas con RP. En esta versión hay que elegir una frase entre un conjunto de cuatro alternativas, ordenadas por su gravedad. Cada ítem se valora con diferentes opciones de respuesta de 0 a 4 , dando una puntuación total posible de 39 puntos. Se toman en consideración las siguientes puntuaciones: 0-4 Depresión Ausente, 5-7 Depresión leve, 8-15 Depresión moderada y $>15$ Depresión grave (Joffre, Martínez, García \& Sánchez, 2007).

\section{Resultados}

Las puntuaciones medias, correspondientes a las variables de ansiedad (estado y rasgo) y depresión, observadas en cada uno de los grupos se incluyen en la Tabla 2.

En primer lugar, el análisis se centró en comparar las puntuaciones de ansiedad (estado y rasgo) y depresión de los tres grupos, aplicando un MANOVA, asegurándose el cumplimiento de los supuestos de esta técnica ( $\mathrm{M}$ de Box $=21.89, p$ 
TABLA 2

Puntuaciones medias y desviaciones típicas en ansiedad y depresión de los tres grupos

\begin{tabular}{lccccccccc}
\hline & \multicolumn{2}{c}{ Grupo RP } & \multicolumn{2}{c}{ Grupo familia } & \multicolumn{2}{c}{ Grupo control } \\
\cline { 2 - 10 } & Media & DT & Media & DT & Media & DT & F & P & Potencia \\
\hline AE & 19.56 & 11.813 & 18.14 & 9.046 & 12.44 & 8.232 & 4.71 & 0.011 & 0.776 \\
AR & 21.72 & 9.663 & 19.77 & 10.533 & 15.29 & 9.226 & 3.76 & 0.027 & 0.676 \\
Depresión & 5.1 & 4.210 & 2.50 & 4.728 & 2.06 & 2.752 & 4.85 & 0.010 & 0.788 \\
\hline
\end{tabular}

Fuente: elaboración propia.

$>0.05)$. Los resultados indicaron la existencia de diferencias significativas entre los grupos (Lambda de Wilks $\left.=0.302, F_{3,83}=64.0 ; p<0.001\right)$, mostrando que los grupos difieren en términos globales en las tres medidas: ansiedad estado $\left[F_{2,85}=4.71\right.$; $p<0.05]$, ansiedad rasgo $\left[F_{2,85}=3.76 ; p<0.05\right]$ y depresión $\left[F_{2,85}=4.85 ; p<0.05\right]$ (Tabla 3).

A continuación, las diferencias entre los grupos se estudiaron con pruebas post-hoc (test de Bonferroni) (Tabla 3), constatando que el grupo RP puntuaba más alto en las tres escalas, hallando diferencias significativas con el grupo control en las tres mediciones: ansiedad estado $(p<0.05)$, ansiedad rasgo $(p<0.05)$ y depresión $(p<0.05)$.

En cuanto al grupo de familiares, los estadísticos descriptivos mostraban que las medias de las puntuaciones en ansiedad se acercaban al grupo RP. De hecho, las pruebas post-hoc mostraban que no había diferencias entre estos dos grupos en las medidas de ansiedad $(p>0.05)$. Por el contrario, en cuanto a depresión, la media del grupo de familiares se acerca más al grupo control, pero al comparar la diferencia de medias entre el grupo RP y el de familiares, aunque no resultan estadísticamente

TABLA 3

Pruebas post hoc de los tres grupos en las variables de ansiedad y depresión

\begin{tabular}{|c|c|c|c|c|c|c|c|}
\hline & & & \multirow{2}{*}{$\begin{array}{l}\text { Diferencia de } \\
\text { medias }\end{array}$} & \multirow{2}{*}{ Error típ. } & \multirow{2}{*}{$\mathrm{p}$} & \multicolumn{2}{|c|}{ IC al $95 \%$} \\
\hline & & & & & & L. inferior & L. superior \\
\hline \multirow{3}{*}{$\mathrm{AE}$} & $\mathrm{RP}$ & Familia & 1.43 & 2.734 & 1.000 & -5.25 & 8.10 \\
\hline & Familia & Control & 5.70 & 2.701 & 0.114 & -0.90 & 12.29 \\
\hline & Control & $\mathrm{RP}$ & -7.12 & 2.431 & 0.013 & -13.06 & -1.18 \\
\hline \multirow{3}{*}{ AR } & $\mathrm{RP}$ & Familia & 1.95 & 2.692 & 1.000 & -4.63 & 8.52 \\
\hline & Familia & Control & 4.48 & 2.660 & 0.288 & -2.02 & 10.98 \\
\hline & Control & $\mathrm{RP}$ & -6.42 & 2.394 & 0.026 & -12.27 & -0.58 \\
\hline \multirow{3}{*}{ Depresión } & $\mathrm{RP}$ & Familia & 2.38 & 1.070 & 0.087 & -0.24 & 4.99 \\
\hline & Familia & Control & 0.44 & 1.057 & 1.000 & -2.14 & 3.02 \\
\hline & Control & $\mathrm{RP}$ & -2.82 & 0.952 & 0.012 & -5.14 & -0.49 \\
\hline
\end{tabular}

Nota. AE: Ansiedad estado; AR: Ansiedad rasgo.

Fuente: elaboración propia. 
significativas, se observa que el $p$-valor se acerca al valor crítico $(p=0.08)$.

Si se comparan las puntuaciones entre el grupo de familiares y el grupo control, encontramos de nuevo que, aunque las diferencias no resultan estadísticamente significativas en las puntuaciones de ansiedad estado, el $p$-valor presenta un valor bajo $(\mathrm{AE} p=0.114 ; \mathrm{AR} p=0.288)$. Un mayor tamaño de muestra podría arrojar diferencias entre estos grupos en ansiedad. En la medida de depresión, las diferencias entre el grupo control y el de familia no resultan estadísticamente significativas.

Para analizar la posible relación de la AV en las medidas de ansiedad y depresión en las personas afectadas con RP, se calculó el coeficiente de correlación de Spearman entre estas variables en este grupo (Tabla 4).

Las correlaciones obtenidas fueron negativas aunque todas muy cercanas a cero $(p>0.05)$, por lo que no hemos obtenido evidencia alguna que lleve a pensar que la AV influya en el nivel de ansiedad o depresión de los afectados por RP.

TABLA 4

Correlación de Spearman entre AV y las variables dependientes en el grupo RP

\begin{tabular}{lccc}
\hline & AE & AR & Depresión \\
\hline Coeficiente & -0.101 & -0.168 & -0.097 \\
$p$ & 0.616 & 0.402 & 0.630 \\
\hline
\end{tabular}

Fuente: elaboración propia.

\section{Discusión}

En el presente estudio hemos evaluado, en primer lugar, la depresión y la ansiedad en personas afectadas por RP frente a un grupo de familiares y otro de no afectados. Los resultados muestran que la ansiedad es mayor en personas con RP y sus familiares, como estado y como rasgo, lo que puede deberse a un sentimiento de amenaza o anticipación del daño, por otro lado comprensible debido al carácter degenerativo de la RP. Las comparaciones entre grupos muestran que el grupo RP se aleja respecto del grupo control en las dos medidas de ansiedad. En cuanto a los familiares, se observa que las puntuaciones en ansiedad se acercan al grupo RP, lo que va en la línea de algunos estudios que sugieren que los efectos de una enfermedad crónica y progresiva, como la que nos ocupa, pueden incidir negativamente en el bienestar y clima familiar no solo de los que la padecen, sino de aquellos con los que conviven (Agudelo et al., 2009; Nemshick et al., 1986).

El otro dato derivado de nuestros resultados pone de manifiesto la presencia de depresión leve en los afectados (Joffre et al., 2007), mientras se observa que las puntuaciones del grupo de familiares se acercan al grupo control, obteniendo un p-valor cercano al valor crítico al comparar el grupo de familiares y RP, indicando que el grupo de familiares no parece evidenciar síntomas de depresión.

La RP parece ser un suceso negativo en la vida de los afectados que, indiscutiblemente, puede tener alguna repercusión en su estado emocional y en su visión de sí mismos, del mundo y del futuro, pero los niveles de depresión que presentan (catalogada como leve) sugieren que pueden haberse adaptado a la enfermedad, aunque no a sus consecuencias. De acuerdo con el estudio de Nemshick et al. (1986), el periodo de mayor crisis o estrés se produce durante o inmediatamente después del diagnóstico, lo que podría explicar nuestros resultados, ya que los participantes habían sido diagnosticados, al menos, tres años antes.

Creemos que el malestar psicológico de las personas con RP, que se traduce en unas puntuaciones más altas en ansiedad y depresión, podría estar relacionado con el temor a perder el control sobre su condición visual, ver reducida su autonomía funcional o su sentido de autoeficacia (Horowitz \& Reinhardt, 2000; Mogk et al., 2000; Rundquist, 2004; Wahl et al., 2004; Zeiss et al., 1996). Igualmente, los familiares parecen mostrar cierta inquietud ante el sufrimiento presente y futuro de sus allegados, lo que quizás explique sus puntuaciones en ansiedad.

No hemos encontrado relación entre la AV y los niveles de depresión y ansiedad en las personas con RP, lo que nos lleva a afirmar que no parece tener repercusión en este momento, dato cohe- 
rente con los resultados del estudio de Hahm et al. (2008).

Estamos de acuerdo con otros autores (Augustin et al., 2007; Hahm et al., 2008 Nemshick et al., 1986; Strougo et al., 1997) en la recomendación de evaluar la depresión y la ansiedad tras el diagnóstico de la enfermedad, al considerarse indicadores de la falta de ajuste emocional a la enfermedad crónica (Sánchez et al., 2006). El conocimiento de estas variables permitiría prevenir el riesgo de depresión en estos afectados, pues existen argumentos de que la presencia de un alto número de síntomas depresivos elevan el riesgo de presentarla posteriormente, sobre todo en grupos con especiales características (Vázquez \& Torres, 2007). Los resultados obtenidos permiten sugerir, asimismo, una nueva evaluación transcurrido un tiempo después del diagnóstico, para conocer cómo se hallan estas variables.

Los datos encontrados llevan a concluir que como la RP es una enfermedad para toda la vida, sin opciones actuales de tratamiento médico, el apoyo psicológico es fundamental para reducir el dolor psicológico, prevenir la mayor discapacidad que asocia la depresión (Augustin et al., 2007; Vázquez \& Torres, 2007) y obtener, de este modo, una mejora del funcionamiento visual (Hahm et al., 2008). También los familiares deberían recibir algún tipo de intervención psicológica, para estar en condiciones de enfrentarse a las consecuencias de esta patología. Sus puntuaciones en ansiedad alertan, pues la comparación entre el grupo RP y de familiares arrojan similitudes.

Se puede afirmar que el estudio aporta datos que pueden ser interesantes para la intervención psicológica con esta población, no obstante, tiene varias limitaciones. En primer lugar, no se ha diagnosticado la depresión utilizando los criterios convencionales, sino un cuestionario de autoinforme. Si bien conviene puntualizar que, aún no siendo una herramienta de diagnóstico para la depresión, ha sido confirmado como un buen método de cribado. Otro inconveniente radica en que se ha usado la versión abreviada, y es conocido que a medida que disminuye el número de ítems también lo hace la fiabilidad; no obstante, se debe señalar que se ha comprobado una correlación alta (0.96) entre ambas versiones (Beck et al., 1979). Segundo, se ha evaluado una sola vez, lo que impide averiguar si la depresión era mayor tras el diagnóstico, como asegura Nemshick et al. (1986), o persiste, lo que haría sospechar que podría tratarse de un rasgo de personalidad; en general, las personas que presentan estos rasgos tienen una disposición a la angustia y un punto de vista más negativo de sí mismos y de las circunstancias, siendo más probables los síntomas depresivos como respuesta a la restricción visual (McCrae \& Costa, 1990).

Vale señalar que el tamaño de la muestra es reducido, lo que supone una limitación en la generalización de los resultados. Por otro lado, el carácter intencional del muestreo, debido a que solo han participado personas voluntarias pertenecientes a una asociación de afectados, impide que se pueda saber cómo se encuentran otros afectados. Sin embargo, se ha procurado equilibrar los grupos respecto a las características sociodemográficas, para asegurar la mayor comparabilidad.

\section{Referencias}

Agudelo, D. M., Casadiegos, C. P. \& Sánchez, D. L. (2009). Relación entre esquemas maladaptativos tempranos y características de ansiedad y depresión en estudiantes universitarios. Universitas Psychologica, 8 (1), 87-104.

Augustin, A., Sabel, J. A., Bandello, F., Dardennes, R., Maurel, F., Negrini, C. et al. (2007). Anxiety and depression prevalence in age-related macular degeneration. Investigative Ophtalmology \& Visual Science, 48 (4), 1498-1503.

Beck, A. T. (1987). Cognitive models of depression. Journal of Cognitive Psychotherapy: An International Quarterly, 1, 5-37.

Beck, A. T., Rush, A. J., Shaw, B. F. \& Emery, G. (1979). Cognitive therapy of depression. New York: Guilford Press.

Beck, A. T., Steer, R. A. \& Garbin, M. C. (1988). Psychometric properties of the Beck Depression Inventory: Twenty-five years of evaluation. Clinical Psychology Review, 8, 77-100. 
Cimarolli, V. \& Boerner, K. (2005). Social support and well-being in adults who are visually impaired. Journal of Visual Impairment and Blindness, 99 (9), 521-534.

Fernández, E. (2007). Retinosis pigmentaria: preguntas y respuestas. Elche: Cátedra Bidons Egara.

Geruschat, D. \& Turano, K. (2002). Connecting research on Retinitis Pigmentosa to the practice of orientation and mobility. Journal of Visual Impairment and Blindness, 96, 69-85.

Hahm, B., Shin, Y., Shim, E., Jeon, H., Seo, J., Cheng, H. \& Yu, H. (2008). Depression and the visionrelated quality of life in patients with Retinitis Pigmentosa. British Journal Ophthalmology, 92, 650-654.

Horowitz, A. \& Reinhardt, J. (2000). Mental health issues in visual impairment: Research in depression, disability and rehabilitation. In B. Silverstone, M. Lang, B. Rosenthal \& E. Faye (Eds.), The Lighthouse Handbook on Vision Impairment and Vision Rehabilitation: Vision Rehabilitation (Vol. 2., pp. 1089-1109). New York: Oxford University Press.

Joffre, V. M., Martínez, G., García, G. \& Sánchez, L. (2007). Depresión en estudiantes de medicina. Resultados de la aplicación del inventario de depresión de Beck en su versión de 13 ítems. Revista Argentina de Clínica Neuropsiquiátrica, 14 (1), 86-93.

Kiser A. \& Dagnelie G. (2008). Reported effects of non-traditional treatments and complementary and alternative medicine by retinitis pigmentosa patients. Clinical and Experimental Optometry, 91 (2), 166-176.

Lee, D. J., Gómez-Marín, O. \& Lam, B. L. (2000). Current depression, lifetime history of depression and visual acuity in Hispanic adults. Journal of Visual Impairment and Blindness, 9 (2), 85-96.

López-Ibor, J. J. \& Valdés, M. (2002). DSM-IV-TR. Manual diagnóstico y estadístico de los trastornos mentales. Barcelona: Masson.

López-Justicia, M. D. (2004). Aspectos evolutivos y educativos de la deficiencia visual. La Coruña: Netbiblo.

López-Justicia, M. D. \& Nieto, I. (2006). Self-concept of Spanish young adults with RP. Journal of Visual Impairment and Blindness, 100 (6), 366-370.
López-Justicia, M. D. \& Pichardo, M. C. (2001). Selfconcept and gender in Spanish low-vision adolescents. Journal of Visual Impairment Research, 3 (1), 7-16.

McCrae, R. R. \& Costa, P. T. (1990). Personality in adulthood. New York: The Guilford Press.

Mogk, L., Riddering, A., Dahl, D., Bruce, C. \& Bradford, S. (2000). Depression and function in adults with visual impairments. In C. Stuen, A. Arditti, A. Horowitz, A. Lanb, B. Rosenthat \& K. R. Siedman (Eds.), Vision rehabilitation assessment, intervention and outcomes (pp. 663-665). Lisse, The Netherlands: Swets and Zeitlinger.

Nemshick, L. A., Vernon, M. \& Ludman, F. (1986). The impact of Retinitis Pigmentosa on young adults: Psychological, educational, vocational and social considerations. Journal of Visual Impairment and Blindness, 89, 859-862.

Páez, A. E., Jofré, M. J., Azpiroz, C. R. \& De Bortoli, M. A. (2009). Ansiedad y depresión en pacientes con insuficiencia renal crónica en tratamiento de diálisis. Universitas Psychologica, 8 (1), 117-124.

Reinhardt, J. P. (2001). Effects of positive and negative support received and provided on adaptation to chronic visual impairment. Applied Developmental Science, 5, 76-85.

Rundquist, J. (2004). Low vision rehabilitation of Retinitis Pigmentosa. Journal of Visual Impairment and Blindness, 98, 718-724.

Sacks, S., Wolffe, K. \& Tierney, D. (1998). Lifestyles of students with visual impairments: Preliminary studies of social networks. Exceptional Children, 64 (4), 463-478.

Sánchez, M. P., Aparicio M. E. \& Dresch, V. (2006). Ansiedad, autoestima y satisfacción autopercibida como predictores de la salud: diferencias entre hombres y mujeres. Psicothema, 18 (3), 584-590.

Sanz, J. \& Vázquez, C. (1998). Fiabilidad, validez y datos normativos del Inventario para la Depresión de Beck. Psicothema, 2, 303-318.

Spielberger, C. D., Gorsuch, R. L. \& Lushene, R. E. (2002). Manual STAI. Cuestionario Ansiedad Estado-Rasgo. Madrid: TEA Ediciones.

Strougo, Z., Badoux, A. \& Duchanel, D. (1997). Problèmes psycho-affectifs associés à la Rétinopathie 
Pigmentaire. Journal Français d'Ophtalmologie, 20, $111-116$

Vázquez, C. \& Sanz, J. (1997). Fiabilidad y valores normativos de la versión española del Inventario de Depresión de Beck 1978. Clínica y Salud, 8, 403-422.

Vázquez, F. \& Torres, A. (2007). Análisis sobre la investigación de la prevención de episodios nuevos de depresión. Clínica y Salud, 18, 221-246.
Wahl, H., Becker, S., Burmedi, D. \& Schilling, O. (2004). The role of primary and secondary control in adaptation to age-related vision loss: A study of older adults with macular degeneration. Psychology and Aging, 19, 235-239.

Zeiss, A. M., Lewinsohn, P. M., Rohde, P. \& Seeley, J. R. (1996). Relationship of physical disease and functional impairment to depression in older people. Psychology and Aging, 11, 572-581. 